\title{
JOT JOURNAL OF TURBULENCE
}

\author{
http://jot.iop.org/
}

\section{The kinematics and dynamics of vorticity in the inertial range: a possible scenario $\dagger$}

\author{
Anthony Leonard \\ Graduate Aeronautical Laboratories, California Institute of Technology, \\ Pasadena, CA 91125, USA \\ E-mail: tony@galcit.caltech.edu
}

Received 31 October 2002

Published 10 December 2002

\begin{abstract}
The evolution of initially weak structures of vorticity as they evolve in an incompressible turbulent flow is investigated. Such objects are candidates for being important structures in the inertial range and in the dissipation range of scales. Initially, these structures evolve passively as a result of the induced velocity field of the large-scale vorticity field. This field is three dimensional and time dependent, so these objects are subjected to straining apropos of Lagrangian chaos, characterized by a distribution of finite-time Lyapunov exponents.

PACS numbers: 47.32.Cc, 47.27.Gs, 47.27.Ak
\end{abstract}

\section{Contents}

1 Introduction 2

2 Kinematics of passive vorticity 2

3 Properties of the deformed blob 3

4 Concluding remarks 5

$\dagger$ This article was chosen from Selected Proceedings of the 4th International Workshop on Vortex Flows and Related Numerical Methods (UC Santa-Barbara, 17-20 March 2002), ed E Meiburg, G H Cottet, A Ghoniem and P Koumoutsakos. 
The kinematics and dynamics of vorticity in the inertial range: a possible scenario

\section{Introduction}

The evolution of weak structures of vorticity as they evolve in an incompressible turbulent flow is investigated. Such objects are candidates for being important structures in the inertial range and in the dissipation range of scales. These structures evolve passively as a result of the induced velocity field of the large-scale vorticity field. This latter field is three dimensional and time dependent, so these objects are subjected to straining apropos of Lagrangian chaos, characterized by a distribution of finite-time Lyapunov exponents. In this paper we consider the deformation of these weak structures and the resulting self-energy spectrum. We show that time-averaging leads to a fractional power law for the energy spectrum. This work follows along lines presented earlier in $[1,2]$.

\section{Kinematics of passive vorticity}

If $\mathcal{U}$ and $\boldsymbol{\Omega}$ are, respectively, the velocity and vorticity fields of the active, large-scale turbulence and $\boldsymbol{u}$ and $\boldsymbol{\omega}$ the fields of the weak, passive turbulence, then the exact momentum equations are

$$
\begin{aligned}
& \frac{\partial \mathcal{U}}{\partial t}+\boldsymbol{\Omega} \times \mathcal{U}+\boldsymbol{\Omega} \times \boldsymbol{u}=-\nabla P+\nu \nabla^{2} \mathcal{U} \\
& \frac{\partial \boldsymbol{u}}{\partial t}+\boldsymbol{\omega} \times \mathcal{U}+\boldsymbol{\omega} \times \boldsymbol{u}=-\nabla p+\nu \nabla^{2} \boldsymbol{u} .
\end{aligned}
$$

Because the passive vorticity is assumed to be relatively weak, the self-induced motions are negligible and we neglect the term $\boldsymbol{\omega} \times \boldsymbol{u}$ in (2). To illustrate the process we assume $\boldsymbol{\omega}$ to be initially a spherical vortex ring with a Gaussian profile:

$$
\boldsymbol{\omega}(\boldsymbol{x}, 0)=\boldsymbol{\nabla} \times\left[\boldsymbol{a}(0) \exp \left(-\left|\boldsymbol{x}-\boldsymbol{x}_{p}(0)\right|^{2} / \sigma^{2}\right)\right]
$$

where $\boldsymbol{x}_{p}(t)$ is the location of the vortex element or blob that moves with velocity $\boldsymbol{U}\left(\boldsymbol{x}_{p}(t), t\right)$. In addition, the blob packet is assumed to remain small relative to spatial variations in $\mathcal{U}$ beyond linear, so

$$
\mathcal{U}_{i}(\boldsymbol{x}, t)=\mathcal{U}_{i}\left(\boldsymbol{x}_{p}(t), t\right)+U_{i, j}(t)\left(x_{j}-x_{p j}\right)
$$

where $U_{i, j}(t)$ is the velocity gradient tensor

$$
U_{i j}(t)=\frac{\partial \mathcal{U}_{i}}{\partial x_{j}}\left(\boldsymbol{x}_{p}(t), t\right) .
$$

Therefore, the vorticity field of the blob will satisfy the following linearized vorticity transport equation:

$$
\frac{\partial \omega_{i}}{\partial t}+U_{j, \ell}\left(x_{\ell}-x_{p \ell}\right) \frac{\partial \omega_{i}}{\partial x_{j}}=\omega_{j} U_{i, j}+\nu \nabla^{2} \omega_{i}
$$

where $U_{j, \ell}$ is a function of time only. As shown in [1], equation (6) can be solved exactly for the blob to give

$\boldsymbol{\omega}(\boldsymbol{x}, t)=\boldsymbol{\nabla} \times\left\{\boldsymbol{a}(t) \sigma^{3}(\operatorname{det} \boldsymbol{M})^{1 / 2} \exp \left[-\left(\boldsymbol{F}^{-1} \boldsymbol{M} \boldsymbol{F}^{\mathrm{T}-1}\right):\left(\boldsymbol{x}-\boldsymbol{x}_{p}(t)\right)\left(\boldsymbol{x}-\boldsymbol{x}_{p}(t)\right)\right]\right\}$

i.e., a compact object, ellipsoidal in shape. Here $\boldsymbol{F}$ is the deformation tensor satisfying

$$
\frac{\mathrm{d} \boldsymbol{F}}{\mathrm{d} t}=\boldsymbol{U} \boldsymbol{F} \quad(\boldsymbol{F}(0)=\boldsymbol{I}) .
$$

The tensor $\boldsymbol{M}$ takes care of viscous effects via

$$
\frac{\mathrm{d} \boldsymbol{M}^{-1}}{\mathrm{~d} t}=4 \nu \boldsymbol{F}^{-1} \boldsymbol{F}^{\mathrm{T}-1} \quad\left(\boldsymbol{M}(0)=\boldsymbol{I} / \sigma^{2}\right)
$$

and the impulse vector is given by

$$
\boldsymbol{a}(t)=\boldsymbol{F}^{\mathrm{T}-1} \boldsymbol{a}(0) .
$$


The kinematics and dynamics of vorticity in the inertial range: a possible scenario

\section{Properties of the deformed blob}

As shown in [1], we can transform (7) to coordinates along the principal directions of $\boldsymbol{F F}^{\mathrm{T}}$ to find

$$
\boldsymbol{\omega}(\tilde{\boldsymbol{x}}, t)=\tilde{\boldsymbol{\nabla}} \times\left\{\tilde{\boldsymbol{a}}(t) \exp \left[-\left(\frac{\tilde{x}_{1}^{2}}{\ell_{1}^{2}}+\frac{\tilde{x}_{2}^{2}}{\ell_{2}^{2}}+\frac{\tilde{x}_{3}^{2}}{\ell_{3}^{2}}\right)\right]\right\} .
$$

Viscous effects are assumed negligible in the $\tilde{x}_{1}$ - and $\tilde{x}_{2}$-directions, so

$$
\ell_{i}^{2}=\sigma^{2} \mathrm{e}^{2 \lambda_{i} t} \quad(i=1,2) .
$$

Here $\mathrm{e}^{2 \lambda_{i} t}(i=1, \ldots, 3)$ are the eigenvalues of $\boldsymbol{F} \boldsymbol{F}^{\mathrm{T}}$ defining the finite-time Lyapunov exponents, $\lambda_{i}=\lambda_{i}\left(\boldsymbol{x}_{p}(0), t\right)$, ordered so that $\lambda_{1} \geq \lambda_{2} \geq \lambda_{3}$. Their sum is zero because of incompressibility. If viscous effects become important, they will first manifest themselves in the $\tilde{x}_{3}$-direction, the direction of maximum compression. Then $\ell_{3}^{2}$ is given approximately as

$$
\ell_{3}^{2} \cong \mathrm{e}^{2 \lambda_{3} t}\left(\sigma^{2}+4 \nu \int_{0}^{t} \mathrm{e}^{-2 \lambda_{3}\left(\boldsymbol{x}_{p}(0), t^{\prime}\right) t^{\prime}} \mathrm{d} t^{\prime}\right)
$$

The self-energy spectrum for the blob is

$$
E_{s}(k, t)=\frac{1}{2} \int|\hat{\boldsymbol{\omega}}|^{2} \mathrm{~d} \boldsymbol{\Omega}_{\boldsymbol{k}}=\frac{1}{2} \int_{0}^{2 \pi} \mathrm{d} \psi \int_{-1}^{+1} \mathrm{~d} \mu|\hat{\boldsymbol{\omega}}|^{2}
$$

where $\hat{\boldsymbol{\omega}}(\boldsymbol{k}, t)$ is the Fourier transform of $\boldsymbol{\omega}(\tilde{\boldsymbol{x}}, t)$. We find that [1], depending on the magnitude of $k \sigma, E_{s}(k, t)$ is given approximately as follows:

(i) small $k \sigma\left(k \sigma \ll \mathrm{e}^{-\lambda_{1} t}\right)$ :

$$
E_{s}(k, t) \approx \frac{\pi \sigma^{4} \hat{a}_{3}^{2}}{6} \mathrm{e}^{2\left(\lambda_{1}+\lambda_{2}\right) t}(k \sigma)^{2}
$$

(ii) intermediate $k \sigma\left(\mathrm{e}^{-\lambda_{1} t} \ll k \sigma \ll \mathrm{e}^{-\lambda_{2} t}\right)$ :

$$
E_{s}(k, t) \approx \frac{\sqrt{2} \pi^{3 / 2} \sigma^{4} \hat{a}_{3}^{2}}{16} \mathrm{e}^{\left(\lambda_{1}+2 \lambda_{2}\right) t}(k \sigma) ;
$$

(iii) large $k \sigma\left(\mathrm{e}^{-\lambda_{2} t} \ll k \sigma\right)$ :

$$
E_{s}(k, t) \approx \frac{\pi \sigma^{4}}{4} \exp \left[-\frac{k^{2} \sigma^{2} \alpha_{3}(t)}{2} \mathrm{e}^{2 \lambda_{3} t}\right] \mathrm{e}^{\left(\lambda_{1}-\lambda_{2}\right) t}\left[\hat{a}_{3}^{2}(k \sigma)^{-2}+\hat{a}_{2}^{2} \mathrm{e}^{-2\left(\lambda_{1}+\lambda_{2}\right) t}\right]
$$

where $\alpha_{3}(t)$ is defined as

$$
\alpha_{3}(t)=1+\frac{4 \nu}{\sigma^{2}} \int_{0}^{t} \mathrm{e}^{-2 \lambda_{3}\left(\boldsymbol{x}_{p}(0), t^{\prime}\right) t^{\prime}} \mathrm{d} t^{\prime}
$$

and where the $\hat{a}_{i}$ differ from the $a_{i}$ by a rotation.

The small- $k \sigma$ result for $E_{s}$ is seen to be proportional to $(k \sigma)^{2}$ and the square of the impulse as expected. For intermediate values, $E_{s}$ is proportional to $k \sigma$. This is the result that one obtains for two vortex tubes that are nearly parallel to each other, separated by a small distance $d(k d \ll 1)$, and with equal and opposite circulations. For large $k \sigma$ the sheetlike cross-section of the vortex tubes becomes apparent, hence the $(k \sigma)^{-2}$ behaviour above. But also the fact that there are two opposing sheets is manifested by the constant, $(k \sigma)^{0}$, component proportional to $\hat{a}_{2}^{2}$. In the right-hand image of figure 1 , one can find the sheet structure for approximately $\left|\tilde{x}_{2}\right| \geq 2$ and the two overlapping sheets for approximately $\left|\tilde{x}_{2}\right| \leq 1$.

Straining along the direction of maximum compression produces fine scales and thus highwavenumber contributions to the energy spectrum. Viscous effects come into play by limiting 
The kinematics and dynamics of vorticity in the inertial range: a possible scenario
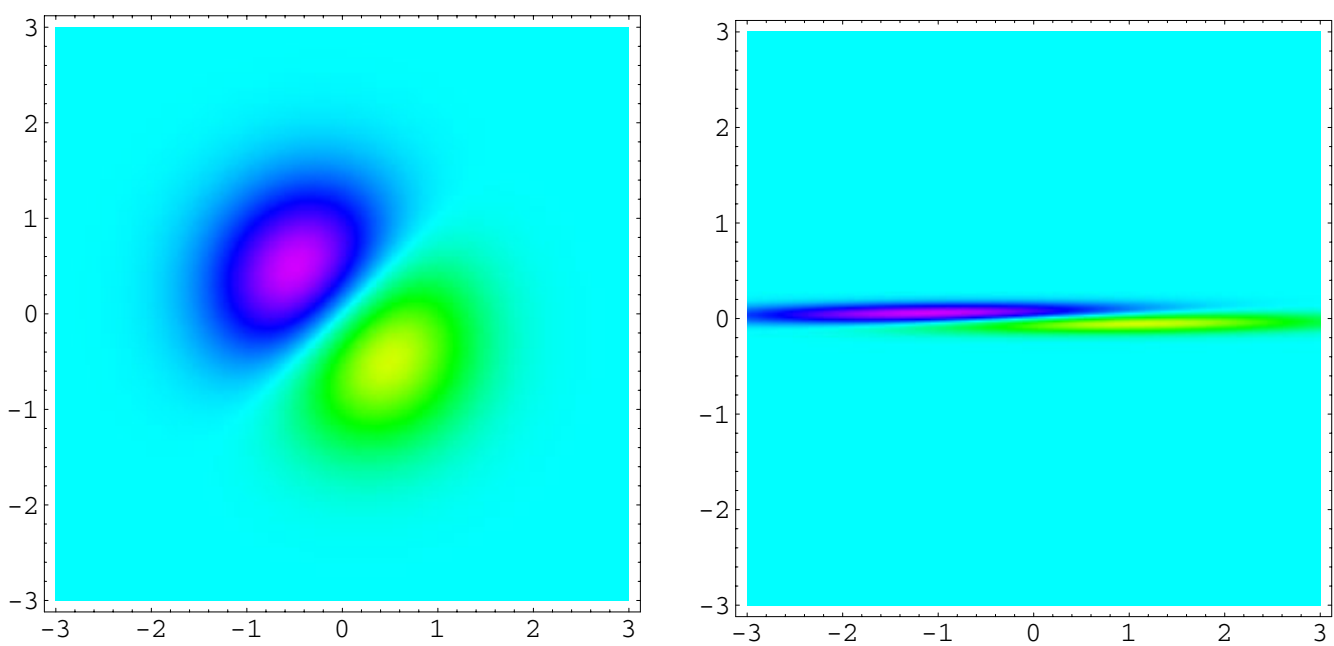

Figure 1. The vorticity component $\mathrm{e}^{-\lambda_{1} t} \omega_{1}$ in the $\left(\tilde{x}_{2}, \tilde{x}_{3}\right)$ plane for the spherical vortex ring initial condition. $\nu=0, \sigma=1, \lambda_{1} / \lambda_{2}=\ln [3 / 2] / \ln [2]=0.5805$. Left: $t=0$. Right: $t=\ln [4] / \lambda_{1}$.

Table 1. Power-law exponents of the energy spectrum for various ratios of the first two Lyapunov exponents.

\begin{tabular}{lll}
\hline Source & Lyapunov exponents & $\bar{E}(k)$ \\
\hline 'Pancakes' & $\lambda_{2}=\lambda_{1}$ & $k^{-2}$ \\
Stretched vortex model $^{\mathrm{a}}$ & $\lambda_{2}=\lambda_{1} / 2$ & $k^{-5 / 3}$ \\
DNS $^{\mathrm{b}}$ & $\lambda_{2}=\lambda_{1} / 7$ & $k^{-10 / 7}$ \\
Reversible dynamics $^{\mathrm{c}}$ & $\lambda_{2}=0$ & $k^{-1}$ \\
'Cigars' & $\lambda_{2}=-\lambda_{1} / 2$ & $k^{1}$ \\
\hline
\end{tabular}

a Reference [4]; see also [5].

b Reference [3].

c Reference [6].

the maximum wavenumber that can be achieved. For a more general initial condition we would expect multiple thin layers of vorticity of alternating sign in the direction of maximum compression. Figure 2 illustrates such a case before viscous effects take place. The compression process proceeds until the layered structure is wiped out by viscous diffusion. This allows us to take a meaningful time average of the self-energy spectrum of the vortex structure. If it is assumed that the $\lambda_{i}$ are constant in time, we find [2] that the time-averaged spectrum has a power-law behaviour with an exponent depending on the ratio of the first two Lyapunov exponents, $\lambda_{2} / \lambda_{1}$, of the strain tensor. As seen in table 1 , if this ratio is $1 / 2$ then the spectrum is Kolmogorov's $k^{-5 / 3}$-law. This result provides an interesting connection to the work of Lundgren [4], who found the same result for the time-averaged spectrum of weak, twodimensional distributions of vorticity in the velocity field of an axisymmetric vortex tube that is being stretched at a constant rate. This result also indicates the importance of obtaining statistics on the strain tensor for turbulent flows (see, e.g., [3]) rather than the more popular object of investigation, the strain-rate tensor. 
The kinematics and dynamics of vorticity in the inertial range: a possible scenario
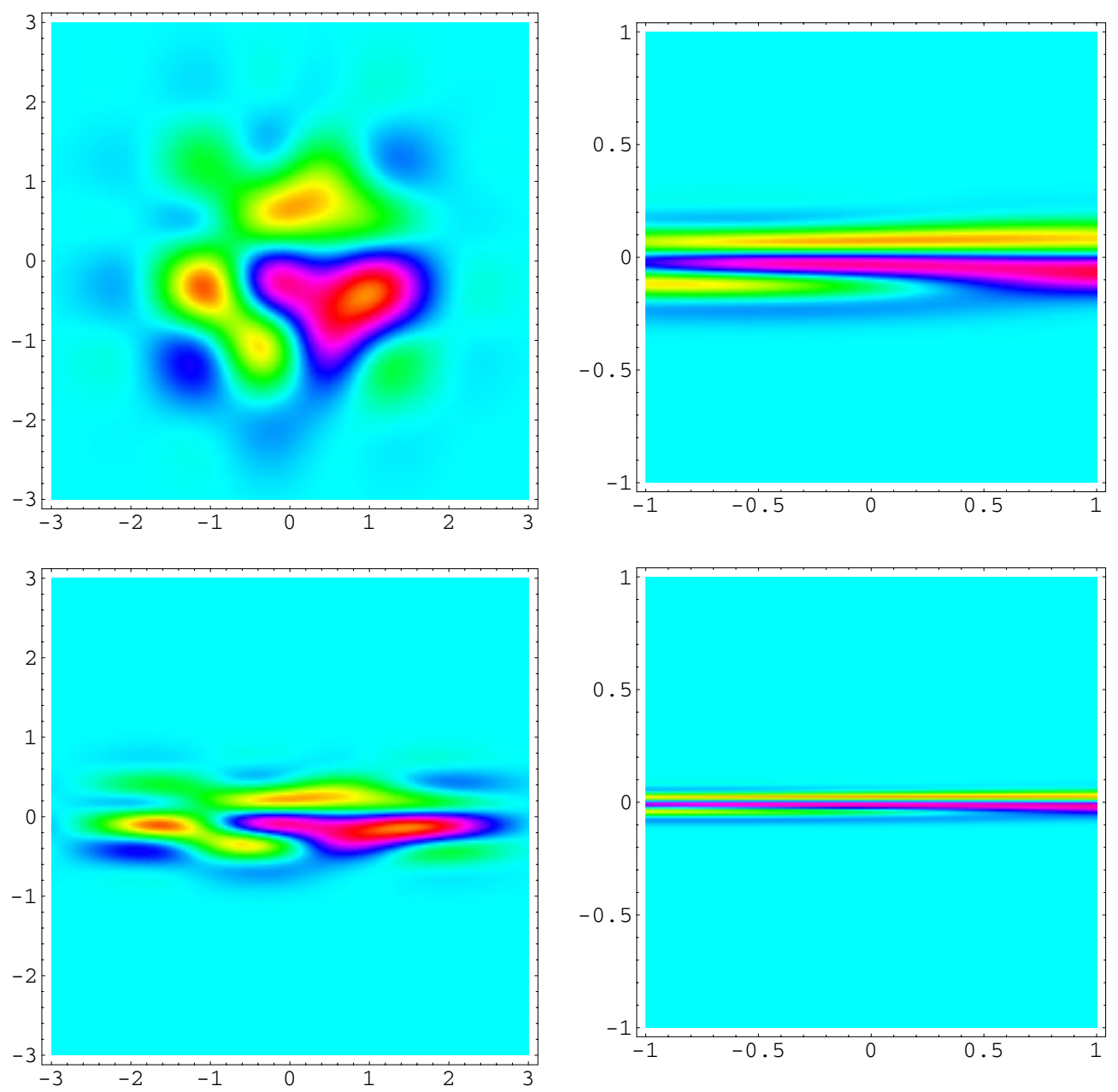

Figure 2. The vorticity component $\mathrm{e}^{-\lambda_{1} t} \omega_{1}$ in the $\left(\tilde{x}_{2}, \tilde{x}_{3}\right)$ plane for a complex initial condition. $\nu=0, \sigma=1, \lambda_{1} / \lambda_{2}=\ln [3 / 2] / \ln [2]=0.5805 \ldots$. Top left: $t=0$. Bottom left: $t=t_{1}=\ln [2] / \lambda_{1}$. Top right: $t=2 t_{1}$. Bottom right: $t=3 t_{1}$. Notice the change in spatial scale for the right-hand figures.

\section{Concluding remarks}

Further work needs to be done to see whether the scenario and related processes described in this paper hold up under further scrutiny and are indeed connected to inertial-range turbulence. For example, time-independent values of Lyapunov exponents should be replaced by ensemble and time averaging over a time-dependent joint probability distribution for $\lambda_{1}$ and $\lambda_{2}$. Further consideration of more general initial vortex structures leading to more complex geometrical shapes as they evolve would be useful. This work should also have implications for subgrid modelling.

\section{References}

[1] Leonard A 2000 Turbulence Structure and Vortex Dynamics (Cambridge: Cambridge University Press) pp 127-39

[2] Leonard A 2002 Interaction of localized packets of vorticity with turbulence Proc. IUTAM Symp. on Tubes, Sheets, and Singularities in Fluid Dynamics (Poland, Sept. 2001) ed K Bajer, at press 
The kinematics and dynamics of vorticity in the inertial range: a possible scenario

[3] Girimaji S S and Pope S B 1990 Material-element deformation in isotropic turbulence J. Fluid Mech. 220 $427-58$

[4] Lundgren T S 1982 Strained spiral vortex model for turbulent fine structure Phys. Fluids 25 2193-203

[5] Gilbert A D 1993 A cascade interpretation of Lundgren's stretched spiral vortex model for turbulent fine structure Phys. Fluids A 5 2831-4

[6] Galluccio S and Vulpiani A 1994 Stretching of material lines and surfaces in systems with Lagrangian chaos Physica A 212 75-98 